\title{
A nova declaração de Helsinque e o uso de placebo em estudos clínicos no Brasil: a polêmica continua
}

\author{
The new declaration of Helsinki and the use of placebo in \\ brazilian clinical trials: controversy remains
}

\author{
Maria Sharmila A. Sousa', Mirian A. G. Franco², João Massud Filho³
}

Sousa MSA, Franco MAG, Massud Filho J. A nova declaração de Helsinque e o uso de placebo em estudos clínicos no Brasil: a polêmica continua / The new declaration of Helsinki and the use of placebo in Brazilian clinical trials: controversy remains. Rev Med (São Paulo). 2012 jul.-set.;91(3):178-88.

RESUMO: Em 2000, dada a urgência de adequação à realidade científica vigente, iniciativas de revisão dos aspectos polêmicos da Declaração de Helsinque $(\mathrm{DoH})$ à publicação de sua quinta versão na qual ficou estabelecido que o uso do placebo seria aceito apenas quando nenhum tratamento existisse. A Federal Drug Administration nos E.U.A., todavia, continuou a exigir controle-placebo, adotando o Guia de Boas Práticas Clínicas. Também a Agência Reguladora Europeia considerou tal proibição arbitrária e, junto a outras entidades, lançaram-se numa campanha contra a DoH. Em 2002 e 2004, Notas de Clarificação publicadas na DoH provocaram grande comoção mundial, culminando na versão de 2008. Apesar da divergência dentre vários grupos, sobretudo, quanto ao uso do placebo, o texto atual assumiu uma posição neutra, flexibilizando sua aplicação. Todavia, uma vez norma ética, a atual $\mathrm{DoH}$ fomentou o reinício das discussões sobre a necessidade de um documento mais ampla e uniformemente aceito. Hoje, esta polêmica ainda continua em alguns países, principalmente no Brasil, onde o Conselho Federal de Medicina (CFM) e a Comissão Nacional de Ética em Pesquisa (CONEP) proíbem o uso de placebo em estudos clínicos em território nacional, quando existir qualquer tratamento disponível. Este fato eliciou grande polêmica entre agências regulatórias e pesquisadores brasileiros. Assim, dado que, desde Nuremberg, a garantia do não enfraquecimento das normas de proteção dos sujeitos de pesquisa recrutados mundo afora é razão primordial de ser da DoH, a reabertura de diálogo em níveis nacional e internacional se faz premente perante a nova revisão comemorativa de seus 50 anos em 2014.

DESCRITORES: Declaração de Helsinki; Placebos; Sociedades médicas/ética; Sociedades médicas/legislação \& jurisprudência; Estudos clínicos como assunto/ética; Brasil.
ABSTRACT: In 2000, given the urgency for the ethical guidelines adequacy to the present scientific standards, revision initiatives of the most controversial aspects of the Declaration of Helsinki (DoH). These efforts culminated with the publication of its fifth version stating that the use of placebo was acceptable when proven treatment does not exist. The US Federal Drug Administration, however, continued demanding the placebo control and adopted the Good Clinical Practice Guidelines. The European regulatory agency also considered such prohibition arbitrary and, amongst other entities, started a campaign against the DoH. In 2002 and 2004, Clarification Notes published on the $\mathrm{DoH}$ promoted extensive controversy worldwide, concluded at its version of 2008. Despite the divergence amongst many groups, especially on placebo use, the current text assumed a more neutral positioning, flexibilising its application. Being an ethical norm, the current DoH re-started the discussions about the necessity of a document of uniform worldwide acceptance. Today, the controversy still remains in a few countries, especially in Brazil, where governmental regulatory agencies - namely the Federal Council of Medicine and the National Commission for Research Ethics - posed a ban on the placebo use in clinical trials nationally, when there is available treatment, corroborating with the ongoing controversy amongst regulatory agencies and researchers in Brazil. Therefore, since Nuremberg, given the warranty of not weakening the protection of research subjects recruited throughout the world constitutes the $\mathrm{DoH}$ most desired aim, dialogue must be resumed both nationally and internationally, considering the upcoming revision in 2014, at the DoH 50 th anniversary.

KEYWORDS: Helsinki declaration; Placebos; Societies, medical/ ethics; Societies, medical/legislation \& jurisprudence; Clinical trails as topic/ethics; Brasil.

Laboratório de Endocrinologia Molecular e Translacional, Disciplina de Endocrinologia, Departamento de Medicina, Escola Paulista de Medicina, Universidade Federal de São Paulo, São Paulo, SP.

Apresentação oral deste trabalho por Sousa, MSAำ na Reunião Satélite da Associação Médica Mundial durante o 11ํㅡㄹ Congresso Mundial de Bioética realizado em Roterdã, em 26 de junho de 2012.

${ }^{1}$ MSc Medicine, Science \& Society, Social Sciences \& Public Policy School, King's College London, University of London; Mestre em Ciências, Disciplina de Endocrinologia, Departamento de Medicina, Escola Paulista de Medicina, Especialista em Medicina Farmacêutica, Programa de Extensão Acadêmica, e Membro-Relatora do Comitê de Ética em Pesquisa, Universidade Federal de São Paulo.

${ }^{2}$ Coordenadora da Comissão de Ética no Uso de Animais (CEUA) do Instituto Dante Pazzanese de Cardiologia, Especialista em Bioética, Instituto Oscar Freire, Faculdade de Medicina, Universidade de São Paulo, Doutora em Farmacologia, e Assessora Científica do Comitê de Ética em Pesquisa, Universidade Federal de São Paulo.

${ }^{3}$ Presidente da Sociedade Brasileira de Medicina Farmacêutica, Professor e Coordenador do Curso de Especialização em Medicina Farmacêutica, Universidade Federal de São Paulo.

Endereço para correspondência: Maria Sharmila A. Sousa. Rua Pedro de Toledo 669, 11ํandar, Vila Clementino, 04039-032, São Paulo, SP, Brasil. e-mail:sharmila.sousa@gmail.com / sharmila.alina@unifesp.br 


\section{Pesquisa clínica e a declaração de Helsinque - breve histórico}

A prática da medicina como promotora da saúde e do bem estar humano tem sido alvo de regulamentações desde tempos imemoriais. Assim, a busca por um código de conduta para salvaguardar os pacientes é uma premente necessidade sempre atual. Considerando tal premissa, pode-se verificar que desde cerca de 450 a.C. no Corpus Hippocraticum, a observação desta preocupação: "Eu juro que (...) aplicarei os regimes para o bem do doente, segundo o meu poder e entendimento, nunca para causar dano ou mal a alguém. A ninguém darei por comprazer, nem remédio mortal nem um conselho que induza a perda. Do mesmo modo não darei a nenhuma mulher uma substância abortiva”'. Já mais tarde, em 1865, Claude Bernard também afirmava que "o princípio da moralidade médica e cirúrgica consiste em nunca realizar experimentos no ser humano que possa prejudicá-lo, mesmo que o resultado seja vantajoso para a ciência"1.

Todavia, o período que abrange a segunda grande guerra mundial (1939-1945) protagonizou grandes perdas do ponto de vista humano, não somente nos campos de batalha, mas também nos campos de concentração. Mein Kampf (1925), ou "Minha Luta", o livro no qual Adolf Hitler propagou sua teoria sobre eugenia racial, constitui solo fértil para o embasamento doutrinário que permeou os praticantes de experimentação humana durante o nazismo. Na realidade, não só a Alemanha, mas boa parte do mundo ocidental aceitou as teorias veiculadas pela eugenia, matéria cujo intuito era utilizar princípios genéticos para o aperfeiçoamento anatomofisiológico dos seres humanos. A primeira legislação eugênica foi adotada no Canton de Vaud, Suíça. Entretanto, quando da sua adoção na Alemanha, vinte estados dos E.U.A. já possuíam legislações de algum modo dotadas de características eugênicas ${ }^{1}$.

Simultaneamente ao final da guerra, ocorreu o Tribunal de Nuremberg, em 1946, com a publicação do "Código de Nuremberg"1, no qual estão descritas as recomendações para a condução de pesquisas em seres humanos. Em 1964, a Associação Médica Mundial (AMM) revisou tal documento, ampliando-o naquilo que passou a ser denominado "Declaração de Helsinque"2 (na sigla DoH no inglês, "Declaration of Helsinki"), a qual manteve esse nome, mesmo após revisões realizadas em diferentes localidades: Tóquio (1975); Veneza (1983); Hong Kong (1989), Somerset West (África do Sul - 1996); Edimburgo (2000), Tóquio (2004) e mais recentemente em Seul (2008). Na atualidade, a versão de 2008 passou a ser a única versão oficial, substituindo todas as ante- riores, as quais passaram a ser consideradas como documentos históricos².

A partir da década de 1980, no entanto, além da $\mathrm{DoH}$, cujo controverso processo de revisão que culminou em sua última versão de 2008 foi a principal razão pela qual se propôs este texto, surgiram outros documentos com igual utilidade - descrever um conjunto de normas e regulamentações a serem consideradas pelos países signatários de tais códigos para a delimitação das formas metodológica e eticamente corretas para a condução de estudos clínicos em seus respectivos países em determinado tempo histórico. Deste modo, em 1981, as "Diretrizes Internacionais Propostas para a Pesquisa Biomédica em Seres Humanos" foram elaboradas pela Organização Mundial de Saúde (OMS) e pelo Conselho das Organizações Internacionais de Ciências Médicas (CIOMS), com atualizações em 1993 e $2002^{3}$. Em seguida, a OMS editou em 1995 o "Guia de Boas Práticas Clínicas" (da sigla GCP no inglês, "Guidelines for Good Practice for Trials on Pharmaceuthical Products") desenvolvidas durante a ICH (no inglês, "International Conference on Harmonisation of Technical Requirements for Registration of Pharmaceuticals for Human Use') ${ }^{4}$.

Entretanto, tais regulamentações internacionais nasceram de um contexto que visou legislar sobre esta mesma atividade, partindo de um referencial alternativo ao da DoH - buscava-se viabilizar a expansão da produção científico-industrial de fármacos em nível internacional, simultaneamente aproveitando os coloridos locais sobre doenças negligenciadas e atendendo as necessidades éticas de cada região, as quais detinham (ou não) regulamentações locais sobre pesquisa envolvendo seres humanos. Logo, nesta evolução das regulamentações sobre pesquisa clínica, muitos temas despontaram como polêmicos dentro deste ramo da filosofia aplicada, hoje conhecido como "Bioética", após a publicação do oncologista Potter de "Bioethics: a Bridge to the Future" em 1971.

Dentre tal controverso debate, podemos citar os contemporâneos argumentos sobre: a) acesso a medicamentos pós-estudo devido às possíveis repercussões ao andamento clínico dos pacientes submetidos a um protocolo clínico, os quais deixariam de obter seu tratamento e reais condições de melhora frente ao novo fármaco; todavia, com a preocupação com os possíveis efeitos adversos do uso crônico de tal novo agente terapêutico; b) estudo com populações específicas como indígenas, crianças e adolescentes, idosos, grávidas e doentes mentais, os quais apresentam um viés de autonomia devido a suas respectivas incapacidades sociais e dificuldades frente a seu contexto; c) pesquisas com células-tronco embrionárias e a panaceia a elas asso- 
ciadas, devido a seu grande potencial como fonte de cura e de conhecimento das doenças para as quais a ciência ainda não encontrou respostas; d) e o uso do placebo-controle em pesquisas clínicas ${ }^{2}$.

Assim, não obstante a aspiração da AMM em contemplar os parâmetros de forma mais adequada e condizente à atual realidade da pesquisa desenvolvida simultaneamente em nível global, tal objetivo acabou caindo por terra com o polêmico processo de revisões da DoH entre 2000 e 2008, cuja última versão de Seul acabou por flexibilizar os parâmetros éticos para a condução de estudos clínicos mundialmente, segundo alguns autores, principalmente, pelo grupo brasileiro ${ }^{5}$. Isto trouxe à tona a discussão sobre a necessidade de um documento sobre conduta de pesquisas biomédicas em seres humanos com força para ser mais ampla e uniformemente aceito em âmbito mundial, para que não ocorra um enfraquecimento das normas de proteção aos pacientes voluntários de pesquisa recrutados pelo mundo, objetivo primordial e razão de ser da DoH.

Discutiremos aqui, portanto, mais a fundo sobre a questão do uso do placebo em estudos clínicos nos contextos brasileiro e internacional, delineando tal conturbado processo de revisão deste documento que é considerado propriedade de toda a humanidade.

\section{Placebo - usos e críticas}

Apesar de a definição de placebo ser, segundo Shapiro ${ }^{7}$, algo usado como controle em estudos de experimentos, uma substância ou procedimento destituído de atividade para a condição a ser avaliada, desde tempos imemoriais, ele tem sido considerado tratamento realmente eficaz ${ }^{6}$. Em verdade, a complexidade de todos os sistemas envolvidos nas possíveis respostas apresentadas pelo organismo quando em contato com substâncias definidas como placebo, e seu efeito no sistema límbico acrescido do elevado número de possíveis variáveis relacionadas, torna o estudo desse tema extremamente difícil'.

Desta maneira, com o Harris-Kefauver Amendment em 1962, determinou-se, pela primeira vez, a necessidade de utilização de metodologia científica rígida, com o uso de ensaios clínicos, randomizados, controlados por placebo e duplocegos. Esta importância da possibilidade de comparação com um controle, de preferência placebo, faz-se premente em pesquisa clínica sobre determinadas doenças, especialmente nas quais há forte conotação psicossomática, como as neurológicas, as psiquiátricas, as do sistema imune, por exemplo. Isto ficou claro quando, a partir de um estudo realizado em 1927com trabalhadores do bairro
Hawthorne de Chicago nos E.U.A., foi possível inferir que os resultados deste ensaio clínico de verificação da eficácia e segurança do fármaco em questão mantiveram influência positiva pela consciência dos participantes de estarem sendo observados ${ }^{8}$.

Observando tais considerações acerca do placebo, portanto, ponderamos sobre as possíveis situações neste debate sobre seu uso em estudos clínicos, um dos objetivos específicos deste trabalho. Logo, primeiramente, fica claro que o uso de placebocontrole É eticamente aceitável em casos como o de um estudo clínico de doenças para as quais ainda não existem diretrizes claras para o tratamento e prevenção deliberadas por associações médicas mundiais e/ ou pela OMS, como para o tratamento da deficiência de Vitamina $D$, por exemplo. Isto, pois esta é uma doença para a qual a OMS apenas começou a investigar sua prevalência mundial, particularmente na Europa, a partir de 2007, visando, então, decidir se e como deve intervir terapêutica ou preventivamente ${ }^{9}$. Constitui, por conseguinte, estudo clínico oportunamente relevante para o qual a reposição de Vitamina $\mathrm{D}$ ainda não foi preconizada como tratamento padrão-ouro mundial e que, assim, efetivamente se beneficiaria do emprego de um placebo-controle, especialmente em um país localizado próximo à linha do equador, onde a radiação ultravioleta é considerada alta e cuja população apresenta ampla aderência à exposição aos raios solares, como acontece no Brasil.

Por outro lado, a despeito da hegemonia da polêmica de quase 40 anos da 'mentira que cura', conforme Brody em 1977 definiu o efeito placebo, seu valor metodológico foi e tem sido intensamente questionado com base em aspectos éticos e médicos ${ }^{10,11}$. Isto pois ele contraria três dos princípios do Comunicado Belmont, publicado por Beauchamp e Childress em 1979: o de justiça, pois todos os participantes devem ter o direito de acesso ao melhor tratamento possível, com igual razão benefício/risco; o de beneficência, uma vez que se deve não causar danos e deve-se ampliar o número de possíveis benefícios, reduzindo riscos; e o de não-maleficência, já que não é aceitável a possibilidade de que um voluntário possa, de modo previsto, vir a ser lesado no ensaio clínico. Logo, a história vem constantemente nos demonstrando, mesmo após a publicação da DoH, episódios tais como Tuskegee entre 1932 e 19725,12 e a contaminação transversal de HIV na África em $1994^{5,13}$, sobre quando o uso do placebo é definitivamente NÃO aceitável. Assim, exemplos como os mencionados acima reforçam a importância de se contar com um documento forte, como o é a DoH, harmonizando todos os possíveis dilemas éticos sobre a condução de pesquisa clínica utilizando o placebo-controle. 
Os argumentos médicos e de cunho ético contrários ao placebo, versam principalmente sob os seguintes aspectos: a) placebos são farmacologicamente inertes, logo, é repreensível seu uso somente para verificar eventual obtenção de significação estatística; b) atualmente, existe, quase sempre, um tratamento alternativo que é melhor que o emprego do placebo e que poderia ser usado como controle ativo em ensaio clínico; c) ensaios clínicos controlados por placebo frequentemente não descartam a hipótese nula, apresentando defeitos intrínsecos, dado que erros podem acontecer em razão de diversos fatores, tais como dose errada, critérios de inclusão/exclusão errados, número reduzido de pacientes, extensão inadequada de tratamento, caracterização inadequada dos pacientes recrutados, fase e gravidade da doença, entre outros; logo, culminam em conclusões errôneas, colocando os pacientes sob tratamento de placebo em risco e trazendo poucas informações; d) placebos raramente são prescritos deliberadamente na vida real; e) placebo-controles frequentemente não podem manter a verdadeira 'cegueira' para pacientes ou investigadores, particularmente quando o fármaco testado induz efeitos objetivos e subjetivos facilmente mensuráveis, intensos ou muito característicos, como bloqueadores beta-adrenérgicos, anticoagulantes ou antiarrítmicos, por exemplo; f) de acordo com o próprio texto da $\mathrm{DoH}$, é inaceitável que o grupo de participantes em placebo seja colocado em risco de subtratamento para o benefício teórico, em potencial, de um grupo hipotético de pacientes, ou mesmo da população em geral ${ }^{14}$.

Vale ressaltar que, entretanto, sob o ponto de vista clínico, torna-se necessário o uso do placebo, por curto período de tempo, para definir um diagnóstico exato. Tome-se, por exemplo, os casos de hipertensão leve, nos quais é difícil saber se a medição corresponde à realidade ou simplesmente ao efeito do 'jaleco branco'. Nestes casos o uso do placebo, na pesquisa clínica, é muito importante.

Finalmente, uma terceira opção é quando o emprego de um placebo-controle em estudos clínicos MUDA o estado-da-arte sobre o conhecimento científico de uma área, transformando uma intervenção clínica padrão-ouro em tratamento obsoleto e não adequado. A história claramente nos demonstrou esta possibilidade no famoso estudo CAST (no inglês, Cardiac Antiarrtyhmia Suppression Trial), quando entre 1986 e 1992 pacientes alocados no grupo placebo apresentaram menor mortalidade do que aqueles sob uso de fármacos experimental e padrão-ouro na época, mudando as diretrizes do tratamento com antiarrítmicos mundialmente ${ }^{15-20}$. Neste caso, se não houvesse o uso do placebo não se chegaria à conclusão sobre o uso indiscriminado de antiarrítmicos. Provavelmente, entretanto, este protocolo não seria aprovado hoje pelos Comitês de Ética no Brasil, uma vez que houve a indicação de placebo para pacientes com arritmia.

Portanto, o objetivo deste trabalho é o de contextualizar o debate sobre o polêmico processo de transformação regulatório internacional e brasileiro através das revisões da $\mathrm{DoH}$, argumentando que o uso do placebo em estudos clínicos deve constituir questão a ser considerada 'caso-a-caso', sem radicalismos de proibição e/ou permissão plena e cega. Logo, para melhor compreendermos o arcabouço político e regulamentar da $\mathrm{DoH}$, retomemos, primeiramente, a evolução histórica de sua formulação textual quanto ao uso de placebo-controle.

\section{Declaração de Helsinque - revisões e contextualização da polêmica mundial}

A DoH ainda é o documento mais abrangente sobre ética em pesquisa com seres humanos em nível mundial ${ }^{21}$. Entretanto, dado que constitui documento de cunho bioético e, como lhe é característico, deve-se atentar para a sua contemporaneidade (tempo e espaço) com a elaboração permanente de novas revisões para sua contextualização às novas tecnologias, polêmicas e padrões socioculturais e moralmente aceitos de nossas sociedades para as quais será aplicada.

Em sua versão original de 1964, a DoH foi desenvolvida para ampliar e explicar o contexto condicionado pelo Código de Nuremberg. Resultou, então, em um conjunto de normas de conduta mais detalhada sobre pesquisa biomédica, contemplando, em especial, o consentimento voluntário como obrigatório ${ }^{22}$. Já na versão seguinte de Tóquio (1975), apresentou o novo conceito de análise dos protocolos dos ensaios clínicos por comitês de ética independentes, os quais deveriam ser governados pelas leis vigentes em cada país. Em Veneza (1983), a segunda versão da DoH incluiu que a solicitação de consentimento obtido de menores de idade seria uma possibilidade, enquanto que a terceira revisão de Hong Kong (1989) lidou mais com a função e estrutura dos comitês de ética independentes.

A quarta revisão da DoH realizada em Somerset West (República da África do Sul - 1996) apresentou mudanças mais substanciais, principalmente devido ao contexto no qual se encontrava - o de um estudo placebo-controlado sobre a transmissão vertical de HIV em mulheres grávidas africanas publicado em $1994^{13}$, e dentro do contexto de diretrizes alternativas para a ética condução de pesquisa clínica, como as GCP, anteriormente mencionadas. Assim, a despeito da vantagem metodológica do uso do placebo, Peter 
Lurie e Sidney Wolfe publicaram um artigo no New England Journal of Medicine em $1997^{23}$, criticando tal estudo, pois algumas mulheres deram à luz a crianças infectadas por HIV cuja doença poderia ter sido prevenida pelos investigadores ${ }^{5,6,23}$. Este fato causou extrema consternação em muitas pessoas ao redor do mundo e assim, iniciaram-se as polêmicas sobre a eticidade do uso de placebo em ensaios clínicos frente à prática médica assistencial.

Logo, esta revisão de 1996 foi uma das mais significativas, pois adicionou ao parágrafo II.3 a frase: "Isto não exclui o uso de inertes placebos em estudos onde nenhum método diagnóstico ou terapêutico comprovado exista". Este fato levou a FDA a ignorar as revisões subsequentes da $\mathrm{DoH}$, citando apenas esta de 1996, a qual correspondia a sua visão sobre o uso de placebos, pois sempre preferiu demonstrar a superioridade de uma droga frente a placebo em estudos de equivalência. A agência norte-americana explica que tais estudos requerem mais pacientes $\mathrm{e}$, consequentemente, mais tempo e dinheiro; além do fato de que se a nova droga não for tão boa quanto sua antecessora, os médicos prescreverão a antiga. Adicionalmente, a FDA também passou a praticar a lei do 'dois pesos, duas medidas', justificando o uso do placebo uma vez que, em pesquisas fora dos E.U.A., pacientes de países pobres já não teriam mesmo o acesso a nenhum tipo dos tratamentos considerados padrão existentes no país-sede do estudo, caso não estivessem participando do estudo ${ }^{5,6}$.

Desde a publicação da versão de 1996, a polêmica devida a tais eventos continuou, então, sobre dois pontos principais: o padrão de cuidado eticamente requisitado para os participantes de pesquisas biomédicas; e o uso de placebo em pesquisas biomédicas ${ }^{24}$. Além destes, a permanente preocupação com a formulação, obtenção e cumprimento do termo de consentimento livre e esclarecido continuou premente. Assim, a revisão de Edimburgo (2000) foi caracterizada pelo maior, mais amplo e controverso debate existente na história das revisões da DoH realizada pela $\mathrm{AMM}$ e ainda corrente em um grupo de países liderados pelo Brasil. A justificativa para tal se deve, em parte, ao fato de que a pesquisa biomédica de então já havia expandido seu escopo, quando comparado ao de 1975; logo, necessitava se adequar à contemporaneidade (tempo e espaço) científica-industrial de então.

Dessa maneira, embora a maioria das reuniões sobre as propostas de mudanças tenha falhado em alcançar um consenso, e muitos argumentaram que a DoH deveria permanecer como estava ou minimante alterada, após intensa consulta mundial, a AMM emitiu o texto aprovado em outubro de 2000. Neste, a parte mais controversa desta quinta revisão ficou sob a seção 'Princípios Adicionais', que contemplava os parágrafos 29 e 30 . Como previsto, estes parágrafos são relativos ao debate, ainda atual, sobre pesquisa biomédica internacional, demonstrando que a exploração de populações pobres como meio para um fim seria inaceitável, dado o não-benefício destas pessoas. Neste sentido, a DoH endossou um padrão ético mais universalista.

Logo, embora o parágrafo 29 reafirmasse que o uso de placebo somente seria permitido quando nenhuma intervenção comprovadamente eficaz existisse, esta decisão causou muito protesto, pois os placebos não seriam aceitos onde não houvesse intervenções comprovadamente eficazes disponíveis. Isto significou que, ao escolher um desenho de estudo, os padrões de cuidado do mundo desenvolvido deveriam ser aplicados a qualquer pesquisa realizada em seres humanos, incluindo aquelas conduzidas em países em desenvolvimento, o que foi chamado de 'ortodoxia do controle-ativo' por alguns ${ }^{25,26}$.

Em oposição, Levine ${ }^{27}$, Temple e Ellenberg ${ }^{28}$ descreveram a 'ortodoxia do placebo' sobre a insistência de que placebo-controles seriam cientificamente mais eficazes e justificáveis nos casos em que o risco de dano fosse baixo. Assim, este ponto de vista discutiu que em lugares onde não existem padrões de cuidado, como ocorre em muitos países em desenvolvimento e subdesenvolvidos, ensaios controlados por placebo seriam então apropriados. Neste argumento utilitário ${ }^{29}$, a desvantagem de alguns seria justificável pela vantagem de muitos futuros pacientes, o que contraria o princípio da prevalência dos direitos individuais sobre os da ciência, de pacientes em potencial, ou mesmo da sociedade como um todo. Adicionalmente, tal controvérsia diz respeito grandemente à justiça distributiva, um dos princípios do Comunicado Belmont, e também remete ao conceito de distribuição equitativa de danos da pesquisa. Ambos dão margem a diferentes interpretações da formulação do texto da DoH, principalmente quando se diz tratamento 'melhor disponível', pois não se sabe se o referencial situa-se no contexto global ou local ${ }^{30}$. Ademais, o parágrafo 30 tratou do acesso a medicamentos pós-estudo, outro assunto de justiça distributiva.

Deste modo, devido à falta de consenso mundial decorrente da quinta revisão de 2000 e à possibilidade de interpretações dicotômicas, a AMM publicou Notas de Clarificação. No que tange ao parágrafo 29 de Washington (2002), portanto, mudou-se a formulação do texto, acrescentando as circunstâncias nas quais o placebo seria 'eticamente aceitável', enquanto que, sobre o parágrafo 30 de Tóquio (2004), o cuidado pós-estudo tornou-se algo a ser considerado, porém sem absoluta certeza. 
Ambas, portanto, flexibilizando a norma ética de conduta em pesquisa clínica mundial. De acordo com a visão dos que optaram, a partir de então, por exilar a DoH, negando sua anuência, este argumento significou uma resposta da AMM, principalmente, às pressões da FDA, da European Agency for the Evaluation of Medical Products (EMEA) e de corporações interessadas em afrouxar os padrões éticos internacionais para a condução de pesquisas biomédicas em seres humanos ${ }^{5,6,31}$.

Com efeito, neste ínterim, a FDA permitiu, com as GCP, que a sua indústria farmacêutica conduzisse ensaios clínicos internacionais em que pacientes do grupo-controle fossem tratados com placebo, ao invés do melhor padrão de cuidado médico existente ${ }^{31}$. Subsequentemente, a AMM aprovou a sexta revisão da DoH em Seul (2008), cujas mudanças notadamente importantes quanto ao parágrafo 32 , responsável por lidar com o conturbado assunto dos placebo-controles, corroboraram para cristalizar tal polêmica. A resposta da AMM a tais pressões de contextualização de sua norma ética ao padrão atual de desenvolvimento e produção científicaindustrial farmacêutica foi de que agora a nova intervenção experimental deverá ser testada contra a "comprovadamente melhor intervenção existente exceto quando não existir esta comprovadamente melhor intervenção, ou quando houver razões metodológicas convincentes e cientificamente sólidas para o uso do placebo e sendo que os que receberem o placebo não estarão sujeitos a nenhum risco de dano sério ou irreversível"2.

Por este viés, ponderando sobre o emprego de placebo-controles em estudos clínicos, três pontos suscitaram como eticamente problemáticos principalmente pelo viés terminológico, e foram discutidos em publicação recente ${ }^{32}$. O primeiro é o critério de risco de dano - a ausência de risco de dano seria razão suficientemente ética para caracterizar o uso de placebo no grupo-controle como aceitável, apesar de não vedar que tal decisão seja tomada individualmente, mas em vista de uma análise cautelosa de um ou mais comitês de ética independentes e capacitados, até mesmo de diferentes instituições para tal. Sob esta perspectiva, o CIOMS seria bem mais restritivo do que a DoH de 2008 vigente.

O segundo ponto versa sobre as razões metodológicas convincentes - sob este aspecto a DoH, segundo a análise, estaria pecando do ponto de vista ético pois: a) permite o risco de dano sério e irreversível; b) o conector 'ou' permite que apenas uma das disposições completadas seja razão suficiente para o uso do placebo; c) de acordo com o parágrafo 5 , o interesse individual deve prevalecer ao da ciência ou da sociedade, o que aqui fica claramente preju- dicado ${ }^{32}$.

Por fim, o último ponto argumenta sobre a disponibilidade de tratamento comprovado - deste terceiro aspecto podem advir as seguintes interpretações da versão atual da DoH: não-existência ou não-disponibilidade de tratamento existente. Logo, a solução mais ética seria a substituição pela versão anterior - "onde não houver tratamento" - e a não-disponibilidade de tratamento significaria que o placebo estaria relacionado ao risco de extrapolação de dados de testes realizados em população de uma específica região onde ele é aceito para a população de outra no qual ele não o é, eliminando o princípio de justiça e do melhor disponível em qualquer lugar do mundo ${ }^{32}$.

Esta mudança, segundo os bioeticistas favoráveis à reabertura deste debate ${ }^{5,6,31}$ implicou em cada vez mais pesquisas médicas sendo realizadas em outros países por organizações de pesquisa clínica terceirizadas localmente (da sigla CROs no inglês, Contract Research Organisations), visando muitas vezes mais ao lucro, e podendo significar que considerações éticas são supérfluas quando os sujeitos de pesquisa vivem a meio-mundo de distância dos E.U.A. ${ }^{33}$. A preocupação do grupo de países liderados pelo Brasil é se esta tendência promoverá, por conseguinte, estudos em países em desenvolvimento, tais como Índia, China e mesmo Brasil, ou subdesenvolvidos, como muitos dos países africanos, com a inclusão de baixos custos e, possivelmente, baixos padrões éticos, graças ao incentivo econômico para este afrouxamento, com a captação de novos mercados e investidores, como afirmam alguns autores ${ }^{6}$.

Por outro lado, considerando que setenta por cento das pesquisas clínicas são realizadas em países desenvolvidos, esta é uma afirmação preconceituosa pois acaba excluindo países emergentes, tais como Brasil, Índia, China, México e África do Sul da rota do desenvolvimento industrial da área de novos fármacos ${ }^{34}$. Segundo os pesquisadores brasileiros envolvidos com o desenvolvimento de novas terapias, tanto no contexto industrial quanto acadêmico aliados a CROs, a internacionalização das pesquisas está muito mais relacionada à necessidade de se estudar um novo medicamento em diferentes etnias. Deste modo, não se pode confundir pesquisa clínica conduzida de maneira séria com os lucros das empresas e/ou instituições governamentais envolvidas em tais atividades. Afinal, uma boa ciência também pode ser um bom negócio.

Por fim, o parágrafo 32 ainda adiciona que: "Extremo cuidado deve ser tomado para evitar abuso desta opção"2. Todavia, com as novas regras recentemente adotadas pela FDA, permitindo que protocolos sejam conduzidos em locais onde as leis 
vigentes são contrárias e/ou se sobrepõem à $\mathrm{DoH}$, talvez esta polêmica deixe de ser $^{21}$. Esta análise, portanto, demonstra claramente a necessidade de um padrão de conduta internacionalmente consistente e aceito quanto à demanda conceitual de placebo e sobre a harmonização das regulamentações, já que a DoH corresponde a uma série de princípios, e cada país pode, como descrito anteriormente, estabelecer regras mais rígidas ou mais flexíveis. Retomemos, portanto, a aceitação da DoH no contexto mundial para finalmente delinearmos a polêmica ainda grandemente embasada no Brasil.

Declaração de Helsinque - Documentos e Legislações Internacionais

Embora existam vários documentos e regulamentações sobre ética em pesquisa em seres humanos, é a AMM, com sua DoH que detém a maior aprovação dentre os diversos países signatários de seus princípios de boa conduta ética em ensaios clínicos. Isto pois, como muito bem ressaltado no CIOMS: "a DoH é um documento fundamental no campo da ética em pesquisa biomédica e tem tido considerável influência na formulação de legislações e códigos de conduta internacionais, nacionais e regionais" 35 .

Desta forma, podemos citar inúmeros documentos e regulamentações de diversos países que em algum momento citaram, implícita ou explicitamente, em sua formulação a necessidade de condução de pesquisa em seres humanos com base no texto da DoH. Começando, portanto, pelo "Guia Tripartite Harmonisado" (EUA, Europa e Japão) para as GCP, por exemplo, em sua versão de 1996, afirmava que os ensaios clínicos para produtos farmacêuticos deveriam estar em concordância com a revisão corrente da DoH (1996), o qual constituía o embasamento ético mais aceito então ${ }^{36}$.

A DoH foi referida como texto-base para o documento de considerações éticas em pesquisa de vacinas para prevenção de AIDS em maio de 2000 pela UNAIDS ${ }^{37}$. A diretiva da União Européia para as GCP em 2001 publicou que "as bases aceitas para a condução de ensaios clínicos em seres humanos é fundamentada na proteção dos direitos humanos e na dignidade do ser humano no que diz respeito à aplicação da biologia e medicina, como está refletido então na versão de 1996 da DoH"38. O "Guia de princípios de ética médica" da Associação Médica do Commonwealth, em 1993, indicou que deveriam ser observadas as provisões da DoH vigente $(1989)^{39}$, assim como o fez o Guia Nórdico ${ }^{40}$ e a PAHO (no inglês, Pan-American Health Organisation) ${ }^{41}$.

A França ainda é signatária da DoH até sua versão mais recente e, de acordo com Delfosse ${ }^{42}$, a legislação da Bélgica exige conformidade com a DoH. Além disso, no Canadá, o "Tri-Council Policy
Statement" de 2005 sobre a conduta ética para pesquisa envolvendo seres humanos ${ }^{43}$ faz referência sobre a conduta ética em ensaios clínicos sob a luz da revisão da DoH então vigente. Na Noruega, os termos de referência para conduta ética em pesquisa com seres humanos também segue a revisão vigente da $\mathrm{DoH}^{24}$.

A Argentina, por outro lado, segue o texto da versão de 2004 da DoH como base de conduta na pesquisa clínica, de acordo com sua legislação vigente, 'Regulamentação nํ⒈490' de $2007^{44}$. Também na Austrália, o código de conduta ética em pesquisas envolvendo humanos, de $1999^{45,46}$, afirma que o comitê de ética em pesquisa responsável deve estar satisfeito com o protocolo apresentado sob a luz da DoH. Na Nova Zelândia, o Documento n 3 de 1997 inclui a seção "Declaração de Helsinque", que descreve uma série de nove princípios-guia para a condução de ensaios clínicos por investigadores ${ }^{47}$.

Também as "Nrmas de administração de ensaios clínicos" da China ${ }^{48}$, desde 1999, afirmavam que deviam estar em consonância com a DoH e com o CIOMS (1993) "para justiça, respeito, máximo benefício dos sujeitos de pesquisa, evitando os riscos o quanto possível"49. Da mesma forma, no Japão, a legislação no 28 de 1997 do Ministério da Saúde e Bem-Estar sobre os padrões de conduta em ensaios clínicos de produtos farmacêuticos inclui uma seção 2, na qual descreve que os protocolos devem estar em concordância com os princípios da DoH vigente $^{24}$. Na Índia, a legislação vigente, "Guia Ético para Pesquisa Biomédica com Seres Humanos" de $2006^{50}$, também cita a DoH desde sua seção introdutória, enfatizando a importância deste documento não somente como norma de conduta ética, como historicamente.

Um exemplo único é Israel, pois é um dos poucos países nos quais a DoH, versão de 1975, está incorporada à legislação em vigor, "Regulamentação de Saúde Pública" de 1999. Esta estabelece os comitês de ética como "Comitês de Helsinque", atribuindo máxima importância à $\mathrm{DoH}^{24}$. Em Uganda, a regulamentação vigente de 1997 afirma que está em conformidade com o Código de Nuremberg e a DoH atual; enquanto que a África do Sul faz referência detalhada à DoH de 1989 em sua legislação local ${ }^{51}$.

A Espanha, por outro lado, é um exemplo dos países que não cita a DoH em nenhum momento em sua legislação vigente, "Lei 14 de Investigação Biomédica" de $2007^{52}$. Apesar de seu texto ter muitas formulações que remetem ao da DoH, esta lei cita apenas outras leis espanholas, principalmente a 'Lei Orgânica n 15' de 1999. Enquanto isso, o Reino Unido segue as GCP, cujo guia da versão de 1998 foi a revisão de 1996 da $\mathrm{DoH}^{53}$, apesar disso, algumas 
entidades, individualmente, adotam as GCP em sua versão mais recente de 2002, como o Royal College of Paediatrics and Child Health ${ }^{54}$.

O código federal de regulamentações dos E.U.A., a 'Common Rule', arbitrariamente permaneceu em concordância com a revisão de 1996 da DoH até 2007. Isto pois, em 2008, sua agência regulatória (na sigla FDA no inglês, US. Food and Drug Administration) resolveu que os E.U.A. deixaria de ser signatário da DoH para adotar as GCP, principalmente devido mudanças referentes ao uso de placebo e de acesso a medicamentos pós-estudo entre 2000 e $2008^{6}$. Esta drástica decisão foi tomada uma vez que a FDA alegou que a DoH não era mais um texto confiável, já que é tão fácil e frequentemente mudado $^{12,55}$, e porque seriam mais partidários do uso de placebo ${ }^{6,33}$. Tal posição também foi sustentada pela EMEA por considerar a proibição dos estudos placebo-controlados uma decisão arbitrária e que contraria os interesses de saúde pública.

Por fim, na legislação nacional da Alemanha, por exemplo, não há nenhuma referência explícita à $\mathrm{DoH}$ no que diz respeito a ensaios clínicos de produtos farmacêuticos e medicinais. Entretanto, há referência à primeira revisão de 1975, em Tóquio, no texto oficial das seções 40 e 41 do 'Arzneimittelgesetz' (lei de medicamentos). Ademais, em códigos de conduta ética médica (1997), individualmente, há referências à quinta versão de 1996 da DoH sobre as deliberações dos comitês de ética revisores de projetos de pesquisa biomédica envolvendo seres humanos ${ }^{24}$. Portanto, conforme afirmaram dois especialistas alemães, Deutsch e Taupits ${ }^{56}$, "embora a DoH não seja legalmente um instrumento de união sob o crivo de leis internacionais, sua influência em ética médica e em regulamentações nacionais sobre pesquisa biomédica não pode ser esquecido".

Deste modo, apesar de a melhor definição da importância de tal documento ter sido elaborada durante um fórum brasileiro realizado em 2000, na chamada 'Carta de Brasília' a qual afirmou que "embora a DoH seja de responsabilidade da AMM, este documento deve ser considerado uma propriedade de toda a humanidade" ${ }^{24}$, foram Brasil, Portugal, África do Sul e Uruguai, os países que lideraram uma votação contra a revisão de 2008 em Seul. Abordemos, finalmente, a polêmica ainda grandemente sediada no Brasil.

\section{Declaração de Helsinque - revisão de 2008 e polêmica brasileira}

Neste que se tornou o contexto da 'batalha de Helsinque', e tendo em mente que o potencial para o abuso deve ser eliminado, uma afirmação emitida durante o processo da revisão congrega o espírito atual do grupo de trabalho da AMM responsável por tais mudanças na DoH: "Estamos ansiosos por garantir que nenhuma boa pesquisa ética seja restringida, enquanto que, ao mesmo tempo, estamos determinados a não comprometer os princípios éticos que a profissão médica representa"57.

Retomando o contexto regulatório brasileiro, desta maneira, em 1988, o Conselho Nacional de Saúde (CNS) elaborou uma "Norma sobre ética em pesquisa médica" - a Resolução no 01/88 do CNS. Este documento apresentou tanto os princípios éticos previstos em diretrizes internacionais, como as considerações científicas relacionadas com vigilância sanitária e biossegurança, atualizando a insuficiente legislação do país naquele momento. No entanto, um levantamento do Conselho Federal de Medicina (CFM) mostrou que a edição da Resolução 01/88 teve mínima influência nos centros de pesquisa do país e que uma revisão dessa norma era de necessária urgência. Surgiu então, em 1995, o Grupo Executivo de Trabalho que tinha como intenção principal, além da revisão da Resolução 01/88, a missão de apresentar aos membros do CNS a nova diretriz atualizada e com maior poder de alcance entre os pesquisadores brasileiros.

Em 1996, houve a criação da Resolução $196 / 96^{58}$ cuja parte relevante foi a criação da CONEP (Comissão Nacional de Ética em Pesquisa) e dos CEPs (Comitês de Ética em Pesquisa) com grande caráter bioético. Seu texto não se atém somente à pesquisa biomédica, abrangendo pesquisas que usam seres humanos em qualquer área do conhecimento, e que, por isso, pode ser considerada um marco histórico para a pesquisa clínica no Brasil. Isto, dado que o intuito desta resolução foi o da possibilidade de pleno uso, em um futuro próximo, de toda a potência científica brasileira, dado que, hoje, a realização de projetos de pesquisa clínica no país é vista sob outro ângulo tanto pelos pesquisadores quanto pelas indústrias farmacêuticas, as quais passaram a investir mais tempo e recursos no desenvolvimento de projetos de novos fármacos em nosso país.

Em agosto de 2008, São Paulo sediou um fórum da AMM, no qual a comunidade brasileira pôde ser consultada sobre a nova versão da $\mathrm{DoH}$, como preâmbulo para sua aprovação concluída em outubro de 2008. Os pontos debatidos foram o uso do placebo, acesso ao tratamento após a investigação e pesquisa em crianças. As mudanças ratificadas em Seul foram consideradas uma derrota para o Brasil e para o restante da América Latina, além de Portugal, Espanha e África do Sul, que defendiam um texto mais restritivo que vetasse o uso de placebo nos casos em que existam terapias comprovadamente 
eficazes. Defensores da alteração, no entanto, apontaram que tornar a $\mathrm{DoH}$ muito restritiva prejudicaria o avanço da ciência, uma vez que o placebo seria importante para se verificar, por exemplo, os reais efeitos de drogas em certas doenças, como discutido anteriormente.

Assim, defensores em nível nacional sobre a necessidade conceitual de placebo discutem que, conforme descrito na revisão atual, o texto da DoH (2008) continua em consonância à Resolução 196/96 brasileira, a qual observa em seu item III.3 que o estudo deve "obedecer a metodologia adequada" (item d) e que deve "ter plenamente justificada, quando for o caso, a utilização de placebo, em termos de nãomaleficência e necessidade metodológica" (item f) ${ }^{5}$. Em outras palavras, além de sua justificativa ética, estudos com placebo seriam amplamente justificáveis do ponto de vista metodológico.

Todavia, em contraponto a esta visão favorável ao placebo, a qual é apoiada por inúmeros médicos e pesquisadores brasileiros das mais diversas especialidades representadas na figura da Associação Médica Brasileira (AMB), o CFM liberou a Resolução $\mathrm{n}^{\circ} 1.885 / 2008$ deliberando sobre a proibição do uso de placebo de qualquer natureza em pesquisas médicas envolvendo seres humanos, quando houver tratamento eficaz e efetivo para a doença pesquisada ${ }^{59}$. Dessa maneira, não somente a indústria farmacêutica mas também grande parte dos pesquisadores que dependem do uso do placebo para obter dados seguros e consistentes sobre estudos de novos tratamentos ou intervenções clínicas ficaram de mãos atadas. Logo, este grupo argumenta que a atitude tomada pelo CFM, apoiado pela CONEP, engessou a norma de forma que se o placebo não é ético, tais pesquisas estão, a partir de 23 de outubro de 2008, inviabilizadas, uma vez que não se podem usar dados obtidos de forma antiética para a publicação científica.

Por outro lado, esta não seria a única consequência danosa ao desenvolvimento científico de grande parte das pesquisas conduzidas atualmente, pois a atual formulação da $\mathrm{DoH}$ continua a suscitar a possibilidade de interpretações contraditórias, como descrito anteriormente. A aprovação cega e irrestrita de seus princípios tais como estão dispostos em sua versão corrente também não permite que a busca por tais evidências ocorra de maneira metodologicamente segura e eticamente correta. Todavia, à despeito dessas questões que eram e continuam sendo tão recorrentes na atividade de pesquisa biomédica em seres humanos, tanto o CFM quanto a CONEP mantiveram a posição de proibição do uso do placebo, caso exista tratamento comprovadamente eficaz para a doença em estudo.

\section{Declaração de Helsinque e radicalismos - considerações finais}

Mais do que nunca, portanto, a necessidade de um padrão de conduta conciso e uniformemente aceito no mundo se faz vigente. Um dos maiores contribuintes para tal contexto foram as decisões tomadas pela FDA nos E.U.A. de inicialmente marginalizar a $\mathrm{DoH}, \mathrm{e}$ agora as proibições vigentes no Brasil quanto ao uso de placebo em estudos clínicos. Adicionadas a este fator, as diferentes versões - consideradas pela AMM como inválidas - adotadas por inúmeros países em detrimento à versão atual de 2008 , continuam a invalidar as decisões tomadas nas mais diversas localidades pelo mundo no que diz respeito a um padrão ético de conduta para a atividade de pesquisa biomédica feita em seres humanos.

Por fim, a reverberação destes acontecimentos culminou com a deliberação prévia do CFM às decisões da AMM descritas na vigente $\mathrm{DoH}$ de 2008 sobre a polêmica da utilização do placebo. Alguns criticam as atitudes da AMM e do CFM/CONEP, denominando-as soberba e messianismo pragmático, dada a ignorância das reais razões para tais mudanças por ambas as partes em seus respectivos contextos para audaciosas decisões nos níveis nacional e internacional. Outros aplaudem com orgulho tais decisões pois afirmam demonstrar o caráter ético e de soberania de cada país frente às confusas decisões tomadas por aqueles que teimam em não assumir uma posição coesa, conforme se afirma sobre a AMM como um todo.

Recentemente, nosso grupo de autores foi selecionado pela AMM para apresentar esta discussão, aqui pormenorizada, em sua Reunião Satélite durante o $11^{\circ}$ Congresso Mundial de Bioética, sediado em Roterdã, Países Baixos, no dia 26 de junho de 2012. Durante este evento, ficou claro para o nosso grupo, que representou exclusivamente a visão dos pesquisadores da Escola Paulista de Medicina da Universidade Federal de São Paulo aqui citados, que existe uma divergência nacional entre as posições da AMB, alinhada e signatária da AMM e, consequentemente, de sua $\mathrm{DoH}$, em relação à posição do CFM e CONEP sobre a proibição de todo e qualquer estudo placebo-controlado em território nacional. Além disso, também ficou explicitamente evidente que a AMM parece não estar disposta a abrir diálogo sobre este polêmico assunto, reagindo com descrença sobre nosso argumento de análise caso-a-caso. Manifestaram, neste sentido, contumaz desprezo sobre a posição oficial dos órgãos governamentais reguladores das atividades de pesquisa e assistência clínica no Brasil, ressaltando que o placebo tornou-se uma palavra maldita, principal- 
mente no campo dos militantes contrários ao seu uso no Brasil.

Desta maneira, urge salientar que radicalismos para ambos os lados, seja o de viabilizar uma permissão irrestrita, ou o de proibir cegamente a condução de estudos placebo-controlados no Brasil, são um grande equívoco. Isto, pois, considerando a força histórica da Declaração de Helsinque em não somente harmonizar a ética condução da pesquisa clínica, mas também de proteger os sujeitos de pesquisa por todo o mundo, nossa opinião é que o uso do placebo não deve ser considerado exclusivamente sob o ponto de vista pragmático, tampouco por uma visão estritamente idealista. O uso do placebo deve ser considerado via abordagem caso-a-caso, para que os riscos de se participar em um estudo clínico não sejam superiores aos riscos de não se participar em tais estudos.

Enquanto tais radicalismos prevalecerem, continuamos, portanto, sem um documento, em nível internacional, com força capaz de promover aquilo a que tanto se almejava no pós-segunda guerra mundial: a proteção dos participantes de pesquisa como objetivo primordial. Logo, a reabertura de diálogo se faz premente tanto em nível nacional quanto internacional, perante a nova revisão comemorativa de 50 anos da Declaração de Helsinque, em 2014.

Agradecimentos: aos Professores Dr. José Osmar Medina de Abreu Pestana e Dr. Rui Monteiro de Barros Maciel pelos esclarecedores comentários e engrandecedora convivência em nossas atividades acadêmicas na Escola Paulista de Medicina da Universidade Federal de São Paulo.

\section{REFERÊNCIAS}

1. Nuremberg Code. Trials of war criminals before the Nuremberg Military Tribunals under Control Council Law No. 10 (2). Washington, DC: Government Printing Office; 1949.

2. World Medical Association. Declaration of Helsinki. 2008 [cited 2012 Oct.]. Available from: http://www.wma.net/ en/30publications/10policies/b3/17c.pdf.

3. Council for International Organizations of Medical Sciences CIOMS in collaboration with the World Health Organization. International Ethical Guidelines for Biomedical Research Involving Human Subjects. Geneva, Switzerland; 2002.

4. WHO. Guidelines for Good Clinical Practice (GCP) for Trials on Pharmaceutical Products. Geneva, Switzerland; 1995. p.97-137. (Technical Report Series no. 850).

5. Garrafa V, Lorenzo C. Imperialismo moral e ensaios clínicos multicêntricos em países periféricos. Cad Saúde Pública. 2008;24(10):2219-26.

6. FDA abandons Declaration of Helsinki for international clinical trials. Social Medicine Projects (1st June 2008).

7. Shapiro AK, Shapiro E. The powerful placebo: from acient priest to modern physician. Baltimore: John's Hopkins University Press; 1997.

8. Malani A. Regulation with placebo effects. Harvard Law Rev. 2008;121(5):1-55.

9. WHO. Europe Nutrition. WHO to map vitamin D deficiency in Europe; 2010 [cited 2012 June]. Available from: http://www.euro.who.int/en/what-we-do/healthtopics/disease-prevention/nutrition/news/news/2010/11/ who-to-map-vitamin-d-deficiency-in-europe.

10. Burkhardt R, Kienle G. Controlled clinical trials and medical ethics. Lancet. 1978;2:1356.

11. Lasagna L. Placebos and controlled trials under attack [editorial]. Br J Clin Pharmacol. 1979;15(6):373-4.
12. Camporesi S. The FDA decision to shelve the Helsinki Declaration: ethical considerations European School of Molecular Medicine [cited 2009 Jan.]. Available from: http://www.ecancermedicalscience.com.

13. Connor EM, Sperling RS, Gelber R, et al. Reduction of maternal-infant transmission of human immunodeficiency virus type 1 with zidovudine treatment. Pediatric AIDS Clinical Trials Group Protocol 076 Study Group. N Engl J Med. 1994;331(18):1173-80.

14. Oliveira GG, et al. Ensaios clínicos: princípios e prática. Brasília: Ministério da Saúde, Agência Nacional de Vigilância Sanitária; 2006.

15. Ruskin, JN. The Cardiac Arrythmia Suppression Trial (CAST). N Engl J Med. 1989;321(6):386-8.

16. Capone RJ, et al. Events in the cardiac arrythmia suppression trial: baseline predictors of mortality in placebo-treated patients. J Am Coll Cardiol. 1991;18:1434-8.

17. Epstein $A E$, et al. Events in the cardiac arrythmia suppression trial: mortality in the entire population enrolled. J Am Coll Cardiol. 1991;18:14-9.

18. Greene $\mathrm{HL}$, et al. The cardiac arrythmia suppression trial: first CAST ... then CAST-II. J Am Coll Cardiol. 1992;19: 894-9.

19. Pratt CM, Moyé LA. The cardiac arrhythmia suppression trial - casting suppression in a different light. Circulation. 1995;91:245-7.

20. Goldstein S, et al. Association between ease of suppression of ventricular arrhythmia and survival. Circulation. 1995;91:79-83.

21. Global Bioethics Blog. Declaration of Helsinki redux [2008 Oct. 5]. Available from: http://globalbioethics.blogspot. com.br/2008_10_01_archive.html.

22. Dickens BM. The challenge of equivalent protection. In: 
Ref. 85, at v. II, p. A-1.A-17.

23. Lurie $P$, Wolfe SM. Unethical trials interventions to reduce perinatal transmission of the human immunodeficiency virus in developing countries. N Engl J Med. 1997;337 (12):853-6.

24. Human S, Fluss SS. The World Medical Association's Declaration of Helsinki: historical and contemporary perspectives. 5th ed. WMA; 2001. Available from: http://www.wma.net/en/20activities/10ethics/10helsinki/ draft_historical_contemporary_perspectives.pdf

25. Rothman KJ, Michels KB. The continuing unethical use of placebo controls. N Engl J Med. 1994;331(6):394-8.

26. Freedman B, Weijer C, Glass KC. Placebo orthodoxy in clinical research. I: Empirical and methodological myths. J Law Med Ethics. 1996;24(3):243-51.

27. Levine RJ. Some recent developments in the international guidelines on the ethics of research involving human subjects. An N York Acad Sci. 2000;918:170-8.

28. Temple R, Ellenberg SS. Placebo-controlled trials and active-control trials in the evaluation of new treatments. Part 1: ethical and scientific issues. An Intern Med. 2000;133(6):455-63.

29. Elander, G, Hermerén G. Placebo effect and randomized clinical trials. Theoretical Med. 1995;16(2):171-82.

30. Nuffield Council on Bioethics. The ethics of research related to healthcare in developing countries. 2005.

31. Lurie P, Greco DB. US exceptionalism comes to research ethics. Lancet. 2005;365(9465):1117-9

32. Ehni HJ, Wiesin U. International ethical regulations on placebo-use in clinical trials: a comparative analysis. Bioethics. 2008;22(1):64-74.

33. Trials on trial: the food and drug administration should rethink its rejection of the declaration of Helsinki. Nature. 2008;453(7194):427-8.

34. Associação da Indústria Farmacêutica de Pesquisa. Inovação e pesquisa clínica no Brasil. Interfarma. São Paulo: Formag's Gráfica Editora; 2010. v. 2.

35. International Conference on Harmonisation of Technical Requirements for Registration of Pharmaceuticals for Human Use. ICH Harmonised Tripartite Guideline. Choice of Control Group and Related Issues in Clinical Trials: E 10. 2000. Available from: http://www.ich.org/ fileadmin/Public Web Site/ICH_Products/Guidelines/ Efficacy/E10/Step4/E10_Guideline.pdf

36. Drug Information Association. ICH (Step 5)/CPMP Guideline for Good Clinical Practice. Ambler, PA: The Association; 1997. p.93-7.

37. UNAIDS. Ethical considerations in HIV preventive vaccine research: UNAIDS guidance document. Geneva, UNAIDS; 2000.

38. Official Journal of the European Communities. 2001 May 1st; $n^{\circ}$ L 121, p. 34-44.

39. Commonwealth Medical Association. Medical ethics and human rights. London: The Association; 1994. p.42-3.

40. Nordic Council on Medicines. Good clinical trial practice: Nordic guidelines. Uppsala: The Council; 1989.

41. Pan American Health Organization. PAHO ethical guidelines for research involving human subjects. Washington, DC; 2000.
42. Delfosse M-L. Research committees and the principle of justice: putting ethics and law to the test. In: Weisstub DN, editor. Research on human subjects: ethics, law and social policy. Oxford: Elsevier Science; 1998. p. 286-300.

43. Canadian Institutes of Health Research. Natural Sciences and Engeneering Research Council of Canada. Social Sciences and Humanitites Research Council of Canada. Tri-council policy statement: ethical conduct for research involving humans. Canadá; 1998. (Amendments 2000, 2001 and 2005).

44. Ministerio de Salud. Salud publica. Resolución n¹490/2007.

45. Australia. National Health and Medical Research Council Act $n^{\circ}$ 225/1992. Canberra; 2006. (Amended Act $\left.n^{\circ} 50 / 2006\right)$.

46. National Health and Medical Research Council. National Statement on Ethical Conduct in Research Involving Humans. Canberra: The Council; 1999. See also Gillam L. The new National Statement on Ethical Conduct in Research Involving Humans: a commentary. Monash Bioethics Rev. 2000;19(Suppl):1-13.

47. Health Research Council of New Zealand. Health Research Council Guidelines on Ethics in Health Research: Guidelines and Requirements for Researchers. Wellington: The Council; 1996.

48. National Drug Regulation Administration. Drug Clinical Trial Administration Norms. (1999 Sept 1st)

49. Chinese Ministry of Health. Guidelines on Ethical Review of Medical Research. Promulgated by the Committee on Research Involving Human Subjects of the Ministry of Health. 1998.

50. Indian Council of Medical Research. Ethical Guidelines for Biomedical Research on Human Participants. New Delhi; 2006.

51. South African Medical Council. Guidelines on Ethics for Medical Research. Tygerberg: The Council; 1993.

52. Jefatura del Estado. Ley 14/2007 de Investigación Biomédica; 2007.

53. Royal College of Physicians of London. Guidelines on the practice of ethics committees in medical research involving human subjects. 3rd ed. London: The College; 1996.

54. Royal College of Paediatrics and Child Health: Ethics Advisory Committee. Guidelines for the ethical conduct of medical research involving children. Arch Dis Childhood. 2000;82: 177-82.

55. Wolinski H. The Battle of Helsinki. Nature. EMBO Rep. 2006;(7):670-72.

56. Deutsch E, Taupitz J. Göttingen Report. Freedom and Control of Biomedical Research - the planned revision of the Declaration of Helsinki. Wld Med J. 1999;45:40-1.

57. Ferriman A. WMA agrees to refine changes to Declaration of Helsinki. BMJ. 2001;322(7295):1142.

58. Brasil. Ministério da Saúde. CONEP. Conselho Nacional de Saúde. Resolução n 196/96. Brasília; 1996.

59. Conselho Federal de Medicina. Resolução CFM $n^{\circ}$ 1.885, 23 out. 2008. 